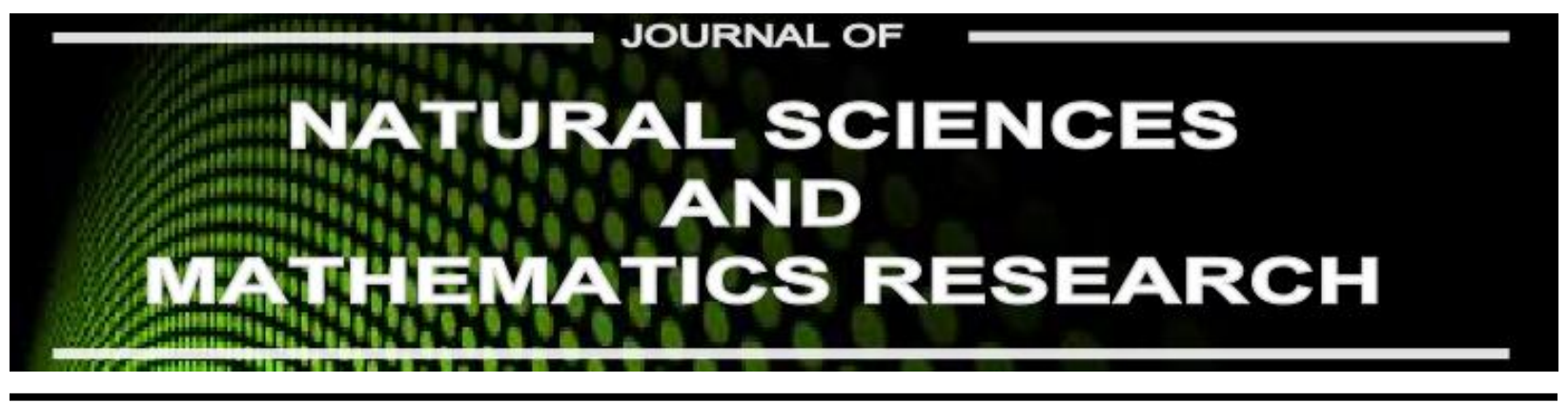

Available online at http://journal.walisongo.ac.id/index.php/jnsmr

\title{
P-Convexity Property in Musielak-Orlicz Function Space of Bohner Type
}

\section{Yulia Romadiastri}

Mathematics Education Departement, Faculty of Sains and Technology, Universitas Islam Negeri Walisongo Central Java,Indonesia

\begin{abstract}
s
Corresponding author: yulia_romadiastri@waliso ngo.ac.id

Recived: 05 May 2017,

Revised : 10 June 2017

Accepted: 30 June 2017.

In this paper, we described about Musielak-Orlicz function spaces of Bochner type. It has been obtained that Musielak-Orlicz function space $L_{\phi}(\mu, X)$ of Bochner type becomes a Banach space. It is described also about P-convexity of Musielak-Orlicz function space $L_{\phi}(\mu, X)$ of Bochner type. It is proved that the Musielak-Orlicz function space $L_{\phi}(\mu, X)$ of Bochner type is P-convex if and only if both spaces $L_{\phi}$ and $X$ are P-convex..(C2017 JNSMR UIN Walisongo. All rights reserved.
\end{abstract}

Key words: Musielak-Orlicz Function; Musielak-Orlicz Functio; Bochner type; PConvex.

\section{Introduction}

The Orlicz space was first introduced in 1931 by W. Orlicz [1]. Orlicz space theory has a very important role and has been widely applied to various branches of mathematics, one of them on the issue of Optimal Control. The development and refinement of the Orlicz space itself is also progressing very rapidly, one them were Musielak and Orlicz [2] which develops a functional space generated by a modular that having convex properties. In this case, $\tilde{I}_{\phi}(f)=\int_{T} \phi\left(t,\|f(t)\|_{X}\right) d \mu$ the modular convex generates the Musielak-Orlicz function spaces of Bochner-type, which is also the Banach space $[3,4]$.

The convexity and reflexive properties of the Banach space also has been widely developed by many mathematicians. Yining, et.al $[5,6]$ in their paper entitled "P-convexity and reflexivity of Orlicz spaces" proved that for Orlicz spaces reflexivity is equivalent to $\mathrm{P}$ convexity. The same result for the MusielakOrlicz sequence and function spaces were obtained by Kolwicz and Pluciennik [7-9]. 


\section{Auxiliary Lemmas}

\section{Definition 1}

Given $\quad f: T \rightarrow \mathbf{R}^{*} \quad$ is $\quad \mu$-measurable function. Function $f$ said to be $\boldsymbol{\mu}$-integrable, if there exist sequence function $\left\{f_{n}\right\}$ such that $f_{n}(x) \rightarrow f(x)$ a.e. $x \in T$ and for every $\varepsilon>0$ there exist natural number $n$ such that $\int_{T}\left|f_{i}(x)-f_{j}(x)\right| \mu(d x)<\varepsilon$, for every $i, j \geq n$.

Hence, the finite value of $\lim _{n \rightarrow \infty} \int_{T} f_{n}(x) \mu(d x)$, is called Lebesgue integral of function $f$ and denoted by $\int_{T} f(x) \mu(d x)$ or $\int_{T} f d \mu$.

Given measurable set $X$. The set of all $\mu$ measurable functions from $X$ to $\mathbf{R}^{*}$ denoted by $M(X)$. It can be proved that $M(X)$ is a linear space. For $1 \leq p<\infty$, defined

$$
L^{p}(X)=\left\{\left.f \in M(X)\left|\int_{X}\right| f\right|^{p} d \mu<\infty\right\} .
$$

In another word, $L^{p}(X)$ is a set of all measurable functions $f \in M(X)$, such that $|f|^{p} \quad \mu$-integrable in $X$.

Given $f$ extended real valued function on measurable set $X$. Supremum essensial $f$ on $E$ defined by

ess sup $\{|f(x)|: x \in X\}=M \Leftrightarrow \exists A \subset X, \mu(A)=0 \ni \sup \{|f(x)|: x \in X-A\}=M$.

Then, we defined $L^{\infty}(X)$ the set of all measurable functions by the formula

$$
L^{\infty}(X)=\{f: \operatorname{ess} \sup \{|f(x): x \in X|\}<\infty\} .
$$

\section{Definition 2}

Given linear space $T$

Non-negatif function $\rho: T \rightarrow[0, \infty)$ is called modular on $T$ if for every $x, y \in T$ this conditions below apply

(M1) $\rho(x)=0 \Leftrightarrow x=\theta$,

(M2) $\rho(-x)=\rho(x)$,
(M3) $\rho(\alpha x+\beta y) \leq \rho(x)+\rho(y)$ if $\alpha, \beta \in[0,1]$ with $\alpha+\beta=1$.

Linear space $T$ that completed with modular is called modular space and denoted by $(T, \rho)$.

A set $B \subseteq Y$, with $Y$ linear space, is called convex set if for each $x, y \in B$ and $\alpha, \beta \in[0,1]$ with $\alpha+\beta=1$, is applicable $\alpha x+\beta y \in B$. Function $f: B \rightarrow \mathbf{R}$ is called convexs, if $B$ convexs and for every $x, y \in B$ and $\alpha, \beta \in[0,1]$ with $\alpha+\beta=1$ be valid $f(\alpha x+\beta y) \leq \alpha f(x)+\beta f(y)$. Furthermore, the modular $\rho$ is called comply character convexs if $\rho$ is fungtion of convexs. On the next discussion, the meaning modular is the modular that comply of character convexs, Unless otherwise stated.

\section{Theorem 3}

(i) If $\alpha_{1}, \alpha_{2} \in \mathbf{R}$ with $0<\alpha_{1} \leq \alpha_{2}$, then $\rho\left(\alpha_{1} x\right) \leq \rho\left(\alpha_{2} x\right)$ for every $x \in X$.

If $\rho(x)<\varepsilon$ for every $\varepsilon>0$, then $x=\theta$.

\section{Definition 4}

Given linear space $T$.

Function $\phi: T \times \mathbf{R} \rightarrow[0, \infty)$ is called Musielak - Orlicz function if:

(1) $\phi(t, u)=0 \Leftrightarrow u=0$, for every $t \in T$,

(2) $\phi(t,-u)=\phi(t, u)$

(3) $\phi(t,$.$) continu$

(4) $\phi(t,$.$) increase on (0, \infty)$

(5) $\phi(\cdot, u)$ measurable, for each $u \in \mathbf{R}$

(6) $\phi(t, \cdot)$ convex,

(7) $\frac{\phi(t, u)}{u} \rightarrow 0$ if $u \rightarrow 0$ on $T$.

For the function of Musielak-Orlicz $\phi$, is defained of function $I_{\phi}: L^{0} \rightarrow[0, \infty)$ with $I_{\phi}(f)=\int_{T} \phi(t, f(t)) d \mu$, 
for every $f \in L^{0}$. So, can be shown that function $I_{\phi}$ is modular convexs. Then, defined space of function Musielak-Orlicz $L_{\phi}$, with $L_{\phi}=\left\{f \in L^{0}: I_{\phi}(c f)<\infty\right.$ for some $\left.c>0\right\}$.

Forthermore, for every of the function Musielak-Orlicz $\phi$, is defained of function $\phi^{*}: T \times \mathbf{R} \rightarrow[0, \infty)$, with

$\phi^{*}(t, v)=\sup _{u>0}\{u|v|-\phi(t, u)\}$,

for every $v \in \mathbf{R}$ and $t \in T$. Can be shown that function $\phi^{*}$ is function of Musielak-Orlicz.

Theorem 5 For every function of MusielakOrlicz $\phi$, be valid $u v \leq \phi(t, u)+\phi^{*}(t, v)$, $u, v \geq 0$, for every $t \in T$.

Definition 6 Function of Musielak - Orlicz $\phi$ is called comply condition $-\Delta_{2}$, writes $\phi \in \Delta_{2}$, if that constanta $k>0$ and $u_{0} \geq 0$ that $\phi(t, 2 u) \leq k \phi(t, u)$, for every $t \in T$ and $u \geq u_{0}$.

Fathermore, defined function $\tilde{I}_{\phi}: L^{0}(T, X) \rightarrow(0, \infty)$ with $\tilde{I}_{\phi}(f)=\int_{T} \phi\left(t,\|f(t)\|_{X}\right) d \mu, \quad$ for every $f \in L^{0}(T, X)$. That, can be shown that function $\tilde{I}_{\phi}$ is modular. For the function of Musielak-Orlicz $\quad \phi, \quad$ defained $L_{\phi}(\mu, X)=\left\{f \in L^{0}(T, X):\|f(.)\|_{X} \in L_{\phi}\right\}$.

The defained $\|\|:. L_{\phi}(\mu, X) \rightarrow \mathbf{R}$, with $\|f\|=\|\| f(.)\left\|_{X}\right\|_{\phi}$, for every $f \in L_{\phi}(\mu, X)$, can be shown that $\left(L_{\phi}(\mu, X),\|\|.\right)$ is normed space.

Theorem 7 Space norm $\left(L_{\phi}(\mu, X),\|\|.\right)$ is space Banach.

Furthermore, the space function $L_{\phi}(\mu, X)$ is called space of function Musielak - Orlicz type Bochner.

\section{Main Result}

Definition 8 Given the space $\operatorname{norm}\left(X,\|.\|_{X}\right)$. The set $S(X)=\left\{x \in X:\|x\|_{X}=1\right\}$ is called area with center $O$ and set $U(X)=\left\{x \in X:\|x\|_{X} \leq 1\right\}$ is called disebut closed unit ball.

Definition 9 The space Banach $X$ is called reflexive if for a $\varepsilon<0$ there $\delta$ so as $\|x-y\|<\varepsilon$ ,for $x, y \in U(X)$ with $\left\|\frac{1}{2}(x+y)\right\|>1-\delta$.

Definition 10 The space norm linear $X$ is called $\boldsymbol{P}$-convexs, if that $\varepsilon>0$ and $n \in N$ so for every $\quad x_{1}, x_{2}, \ldots ., x_{n} \in S(X)$, be valid $\min _{i \neq j ; i, j \leq n}\left\|x_{i}-x_{j}\right\|_{X} \leq 2(1-\varepsilon)$.

Lemma 11 The space Banach $X$ P-convexs if and only if that $n_{0} \in N$ and $\delta_{0}>0$ so for every $x_{1}, x_{2}, \ldots ., x_{n_{0}} \in X \backslash\{0\}$ that integers $i_{0}, j_{0}$ so be valid

$\left\|\frac{x_{i_{0}}-x_{j_{0}}}{2}\right\|_{X} \leq \frac{\left\|x_{i_{0}}\right\|_{X}+\left\|x_{j_{0}}\right\|_{X}}{2}\left(1-\frac{2 \delta_{0} \min \left\{\left\|x_{i_{0}}\right\|_{X},\left\|x_{j_{0}}\right\|_{X}\right\}}{\left\|x_{i_{0}}\right\|_{X}+\left\|x_{j_{0}}\right\|_{X}}\right)$

Theorem 12 To every of function MusielakOrlicz $\phi$ be valid, if $\phi^{*} \in \Delta_{2}$ then for any $a>1$ that $\xi>1$ so $\phi\left(t, \frac{\xi}{a} u\right) \leq \frac{\xi^{-1}}{a} \phi(t, u)$ for every $u \in \mathbf{R}, t \in T$.

Lemma 13 Be discovered $\phi$ and $\phi^{*}$ meet the conditions $\Delta_{2}$.

For every $\varepsilon \in(0,1)$ that of function is measurable $h_{\varepsilon}: T \rightarrow \mathbf{R}^{+}$with $I_{\phi}\left(h_{\varepsilon}\right)<\varepsilon$, number $a(\varepsilon) \in(0,1)$ and $\gamma=\gamma(a(\varepsilon)) \in(0,1)$ such that for $\mu$ h.d. $t \in T$ be valid 
$\phi\left(t, \frac{u+v}{2}\right) \leq \frac{1-\gamma}{2}|\phi(t, u)+\phi(t, v)|$ for $\quad$ every $u \geq h_{\varepsilon}(t)$ and $\left|\frac{v}{u}\right|<a$.

Lemma 14 If $\phi \in \Delta_{2}$, that for every $\alpha \in(0,1)$ there is an undeveloped sequence of measurable sets of up to $\left\{B_{m}^{\alpha}\right\}$, so $\mu\left(T \backslash \bigcup_{m=1}^{\infty} B_{m}^{\alpha}\right)=0$ and for every $m \in N$, there $k_{m}^{\alpha}>2$ so $\phi(t, 2 u) \leq k_{m}^{\alpha} \phi(t, u)$ for $\mu$ a.e., $t \in B_{m}^{\alpha}$ and for every $u \geq \alpha f(t)$, there $f$ of condition $\Delta_{2}$.

.Lemma 15 If $\phi \in \Delta_{2}$, then for every $\varepsilon \in(0,1)$ there is a measurable function $g_{\varepsilon}: T \rightarrow \mathbf{R}^{+}$ and $k_{\varepsilon}>2$ so $I_{\phi}\left(g_{\varepsilon}\right)<\varepsilon \quad$ and $\phi(t, 2 u) \leq k_{\varepsilon} \phi(t, u)$, for $\mu$ a.e., $t \in T$, and $u \geq g_{\varepsilon}(t)$.

Theorem 16 If space Banach X P-convexs, then X reflextif.

Theorem 17 Be discovered $\phi$ function Musielak - Orlicz and X space Banach.

Then the following statements are equivalent:

(a) $L_{\phi}(\mu, X) P$-convex,

(b) $L_{\phi}$ and $X P$-convex,

(c) $L_{\phi}$ reflectif dan $X P$-convex,

(d) X P-convex, $\phi \in \Delta_{2}$ and $\phi^{*} \in \Delta_{2}$.

\section{Evidence:}

$(a) \Rightarrow(b)$

Be discovered $L_{\phi}(\mu, X)$ P-convex. Because of the space $L_{\phi}$ dan $X$ embedded isometrically to $L_{\phi}(\mu, X)$ and characteristics P-convex as well apply on subspace, then obtained $L_{\phi}$ and $X$ P-convex.

$(b) \Rightarrow(c)$
Be discovered $L_{\phi}$ and $X$ P-convex. According to theorem 9 , obtained $L_{\phi}$ reflexive.

$(c) \Rightarrow(d)$

The characteristics of reflexsive on the space funcion of Musielak-Orlicz $L_{\phi}$ ekuivalen with $\phi \in \Delta_{2}$ and $\phi^{*} \in \Delta_{2}$.

$(d) \Rightarrow(a)$

Be discovered $X$ P-convex, $\phi \in \Delta_{2}$ and $\phi^{*} \in \Delta_{2}$. Can be chosen $n_{0} \in N$.

Then, for every $t \in T$ defined $f(t)=\max \left\{\frac{h_{\frac{1}{4 n_{0}}}}{}(t), g_{\frac{1}{4 n_{0}}}(t)\right\}$,

there of function $\frac{h_{\frac{1}{4 n_{0}}}}{\text { and }} g_{\frac{1}{4 n_{0}}}$ is function measureble with $\varepsilon=\frac{1}{4 n_{0}}$.

as a result, $I_{\phi}(f)<\frac{1}{2 n_{0}}$. Can be chosen $\alpha=a$. Because $\phi \in \Delta_{2}$, then obtained $I_{\phi}\left(\frac{1}{a} f\right)<\infty$.

Selected set $\quad B_{m_{0}}^{a} \quad$ so fulfilling $\int_{T \backslash B_{m_{0}}^{a}} \phi\left(t, \frac{f(t)}{a}\right) d \mu<\frac{1}{2 n_{0}}$.

Selected $l \in \mathbf{R}$ so $\frac{1}{a} \leq 2^{l}$. There are numbers $k_{m_{0}}^{a}>2$ so

$\phi\left(t, \frac{1}{a} v\right) \leq\left(k_{m_{0}}^{a}\right)^{l} \phi(t, v)$ for $\quad \mu$-a.e. $t \in B_{m_{0}}^{a}$, when $v \geq a f(t)$.

Then, chosen $\quad \frac{v}{a}=u \quad$ and $\frac{1}{\left(k_{m_{0}}^{a}\right)^{l}}=\beta\left(a, m_{0}\right)=\beta_{m_{0}} . \quad$ Obtained $\phi(t, a u) \geq \beta_{m_{0}} \phi(t, u)$,

For $\mu$-h.d. $t \in B_{m_{0}}^{a}$, and for every $u \geq f(t)$. 
So, obtained $\phi\left(t, \frac{1}{a} v\right) \leq\left(k_{\varepsilon}\right)^{l} \phi(t, v) \quad$ for $\mu$-a.e. $t \in T$, and $v \geq f(t)$, then $k_{\varepsilon}=k_{\frac{1}{4 n_{0}}}$.

In the same way, obtained $\phi(t, a u) \geq \beta \phi(t, u)$,

For $\mu-h . d . t \in T$, and for every $u \geq \frac{f(t)}{a}$.

Furthermore, it will be shown that there are numbers $r_{1} \in(0,1) \quad$ so for every $x_{1}, x_{2}, \ldots, x_{n_{0}}$ space group Banach $X$ and to $\mu$-a.e.t $\in T_{M}$ obtained

$\sum_{i=1}^{n_{0}} \sum_{j=i}^{n_{0}} \phi\left(t,\left\|\frac{x_{i}-x_{j}}{2}\right\|_{X}\right) \leq \frac{n_{0}-1}{2} r_{1} \sum_{i=1}^{n_{0}} \phi\left(t,\left\|x_{i}\right\|_{X}\right)$

With $T_{M}=\left\{t \in T: \max _{1 \leq i \leq n_{0}}\left\{\left\|x_{i}\right\|_{X}\right\} \geq \frac{f(t)}{a}\right\}$.

Taken $x_{1}, x_{2}, \ldots, x_{n_{0}} \in X$. For example $k$ is index so $\left\|x_{k}\right\|_{X}=\max _{1 \leq i \leq n_{0}}\left\{\left\|x_{i}\right\|_{X}\right\}$.

I. Suppose there $i_{1} \in\left\{1,2, \ldots, n_{0}\right\} \backslash\{k\}$ so $\frac{\left\|x_{i_{1}}\right\|_{X}}{\left\|x_{k}\right\|_{X}}<a$.

Because $\quad\left\|x_{k}\right\| \geq \frac{f(t)}{a}>f(t) \quad$ for $\mu-$ a.e.t $\in T_{M}$, so obtained

$\phi\left(t,\left\|\frac{x_{i_{1}}-x_{k}}{2}\right\|_{X}\right) \leq \phi\left(t, \frac{\left\|x_{i_{1}}\right\|_{X}+\left\|x_{k}\right\|_{X}}{2}\right)$

$\leq \frac{1}{2}(1-\gamma)\left(\phi\left(t,\left\|x_{i_{1}}\right\|_{X}\right)+\phi\left(t,\left\|x_{k}\right\|_{X}\right)\right)$.

Based on characteristics convex of $\phi(t,$.$) for$ $\mu$-a.e. $t \in T$, obtained

$\sum_{i=1}^{n_{0}} \sum_{j=i}^{n_{0}} \phi\left(t,\left\|\frac{x_{i}-x_{j}}{2}\right\|_{X}\right) \leq \frac{n_{0}-1}{2} \sum_{i=1}^{n_{0}} \phi\left(t,\left\|x_{i}\right\|_{X}\right)-\frac{\gamma}{2}\left(\phi\left(t,\left\|x_{i}\right\|_{X}\right)+\phi\left(t,\left\|x_{k}\right\|_{X}\right)\right)$

$$
\begin{aligned}
\leq & \frac{n_{0}-1}{2} \sum_{i=1}^{n_{0}} \phi\left(t,\left\|x_{i}\right\|_{X}\right)-\frac{\gamma}{2 n_{0}}\left(n_{0} \phi\left(t,\left\|x_{k}\right\|_{X}\right)\right) \\
& \leq \frac{n_{0}-1}{2} \sum_{i=1}^{n_{0}} \phi\left(t,\left\|x_{i}\right\|_{X}\right)-\frac{\gamma}{2 n_{0}} \sum_{i=1}^{n_{0}} \phi\left(t,\left\|x_{i}\right\|_{X}\right)
\end{aligned}
$$

$$
=\frac{n_{0}-1}{2}\left(1-\frac{\gamma}{n_{0}\left(n_{0}-1\right)}\right) \sum_{i=1}^{n_{0}} \phi\left(t,\left\|x_{i}\right\|_{X}\right),
$$

$$
\text { for } \mu \text {-a.e. } t \in T_{M} \text {. }
$$

II. It is assumed that for all

$$
i \neq k \text { apply } \frac{\left\|x_{i_{1}}\right\|_{X}}{\left\|x_{k}\right\|_{X}} \geq a \text {. }
$$

Then $\left\|x_{i}\right\|>0$, for every $i \neq k$. Can be taken $i_{0}, j_{0}$. and assumed that

$$
a<\frac{\left\|x_{i_{1}}\right\|_{X}}{\left\|x_{j_{0}}\right\|_{X}} \leq \frac{1}{a} .
$$

On the other hand, haved

$a>\frac{\left\|x_{i_{1}}\right\|_{X}}{\left\|x_{j_{0}}\right\|_{X}} \geq \frac{\min \left\{\left\|x_{i_{0}}\right\|_{X},\left\|x_{j_{0}}\right\|_{X}\right\}}{\max \left\{\left\|x_{i_{0}}\right\|\left\|_{X},\right\| x_{j_{0}} \|_{X}\right\}} \geq \frac{\min \left\{\left\|x_{i_{0}}\right\|_{X},\left\|x_{j_{0}}\right\|_{X}\right\}}{\left\|x_{k}\right\|_{X}}$

So it's a contradiction. So obtained

$$
\left\|\frac{x_{i_{0}}-x_{j_{0}}}{2}\right\|_{X} \leq\left(1-\frac{2 \delta a}{1+a}\right) \frac{\left\|x_{i_{0}}\right\|_{X}+\left\|x_{j_{0}}\right\|_{X}}{2} \text {. }
$$

Based on characteristics convex of $\phi(t,$.$) for$ $\mu$-a.e. $t \in T$, obtained

$\phi\left(t,\left\|\frac{x_{i_{0}}-x_{j_{0}}}{2}\right\|_{X}\right) \leq \frac{1}{2}(1-\alpha)\left(\phi\left(t,\left\|x_{i_{0}}\right\|_{X}\right)+\phi\left(t,\left\|x_{j_{0}}\right\|_{X}\right)\right)$

With $\alpha=\frac{2 \delta a}{1+a} \in(0,1)$. so, obtained

$\sum_{i=1}^{n_{0}} \sum_{j=i}^{n_{0}} \phi\left(t,\left\|\frac{x_{i}-x_{j}}{2}\right\|_{X}\right) \leq \frac{n_{0}-1}{2} \sum_{i=1}^{n_{0}} \phi\left(t,\left\|x_{i}\right\|_{X}\right)-\frac{\alpha}{2}\left(\phi\left(t,\left\|x_{i_{0}}\right\|_{X}\right)+\phi\left(t,\left\|x_{j_{0}}\right\|_{X}\right)\right)$ 
$\leq \frac{n_{0}-1}{2} \sum_{i=1}^{n_{0}} \phi\left(t,\left\|x_{i}\right\|_{X}\right)-\frac{\alpha \beta}{n_{0}} \sum_{i=1}^{n_{0}} \phi\left(t,\left\|x_{i}\right\|_{X}\right)$

$=\frac{n_{0}-1}{2}\left(1-\frac{2 \alpha \beta}{n_{0}\left(n_{0}-1\right)}\right) \sum_{i=1}^{n_{0}} \phi\left(t,\left\|x_{i}\right\|_{X}\right)$,

for $\mu$-a.e. $t \in T_{M}$.

Defined

$r_{1}=\max \left\{1-\frac{\gamma}{n_{0}\left(n_{0}-1\right)}, 1-\frac{2 \alpha \beta}{n_{0}\left(n_{0}-1\right)}\right\}$.

Then the number can be determined $r_{2} \in(0,1)$ so be valid $\sum_{i=1}^{n_{0}} \sum_{j=i}^{n_{0}} \phi\left(t,\left\|\frac{x_{i}-x_{j}}{2}\right\|_{X}\right) \leq \frac{n_{0}-1}{2} r_{2} \sum_{i=1}^{n_{0}} \phi\left(t,\left\|x_{i}\right\|_{X}\right)$ , for every $x_{1}, x_{2}, \ldots, x_{n_{0}}$ component space Banach $X$ and for $\mu-h . d . t \in B_{m_{0}}^{a}$ comply maks $_{1 \leq i \leq n_{0}}\left\{\left\|x_{i}\right\|_{X}\right\} \geq f(t)$. Then proved true with

$$
r_{2}=\max \left\{1-\frac{\gamma}{n_{0}\left(n_{0}-1\right)}, 1-\frac{2 \alpha \beta_{m_{0}}}{n_{0}\left(n_{0}-1\right)}\right\} .
$$

Taken $f_{1}, f_{2}, \ldots, f_{n_{0}} \in S\left(L_{\phi}(\mu, X)\right) \quad$ and defained

$$
E=\left\{t \in T: \sum_{i=1}^{n_{0}} \phi\left(t,\left\|f_{i}(t)\right\|_{X}\right) \geq n_{0} \phi(t, f(t))\right\} .
$$

Obviously that, $\max _{1 \leq i \leq n_{0}}\left\{\left\|f_{i}(t)\right\|_{X}\right\} \geq f(t)$ for every $t \in E$. Furthermore $E$ divided into the following two subsets: $E_{1}=\left\{t \in T: \max _{1 \leq i \leq n_{0}}\left\{\left\|f_{i}(t)\right\|_{X}\right\} \geq \frac{f(t)}{a}\right\}$, and $E_{2}=\left\{t \in T: f(t) \leq \max _{1 \leq i \leq n_{0}}\left\{\left\|f_{i}(t)\right\|_{X}\right\}<\frac{f_{i \neq 1}^{n_{0}} t \sum_{j j i}^{n_{0}}}{a} \tilde{I}_{\phi}\left(\frac{1}{2}\left(f_{i}-f_{j}\right)\right)=\sum_{i=1}^{n_{0}} \sum_{j=i}^{n_{0}} \tilde{I}_{\phi}\left(\frac{1}{2}\left(f_{i}-f_{j}\right) \chi_{T \backslash\left(E_{1} \cup E_{21}\right)}\right)+\sum_{i=1}^{n_{0}} \sum_{j=i}^{n_{0}} \tilde{I}_{\phi}\left(\frac{1}{2}\left(f_{i}-f_{j}\right) \chi_{E_{1} \cup E_{21}}\right)\right.$ Defined set $E_{21}$ dan $E_{22}$ as follows $\quad \leq \frac{1}{n_{0}}\left(\begin{array}{c}n_{0} \\ 2\end{array}\right) \sum_{i=1}^{n_{0}} \tilde{I}_{\phi}\left(f_{i} \chi_{T \backslash\left(E_{1} \cup E_{21}\right)}\right)+\frac{r}{n_{0}}\left(\begin{array}{c}n_{0} \\ 2\end{array}\right) \sum_{i=1}^{n_{0}} \tilde{I}_{\phi}\left(f_{i} \chi_{E_{1} \cup E_{21}}\right)$
$E_{21}=E_{2} \cap B_{m_{0}}^{a}$, and $E_{22}=E_{2} \backslash B_{m_{0}}^{a}$.

$$
\sum_{i=1}^{n_{0}} \sum_{j=i}^{n_{0}} \phi\left(t,\left\|\frac{f_{i}(t)-f_{j}(t)}{2}\right\|_{X}\right) \leq \frac{1}{n_{0}}\left(\begin{array}{c}
n_{0} \\
2
\end{array}\right) \sum_{i=1}^{n_{0}} \phi\left(t,\left\|f_{i}(t)\right\|_{X}\right)
$$

for $\mu$-a.e. $t \in E_{1} \cup E_{2}$, with $r=\max \left\{r_{1}, r_{2}\right\}$. obviously $r \in(0,1)$. Furthermore, from the define set $E$ and function $f$, obtained

$$
\sum_{i=1}^{n_{0}} \tilde{I}_{\phi}\left(f_{i} \chi_{T \backslash E}\right)<\frac{1}{2} .
$$

taken $t \in E_{22}$, then obtained

$$
\begin{aligned}
& \sum_{i=1}^{n_{0}} \tilde{I}_{\phi}\left(f_{i} \chi_{E_{22}}\right)=\sum_{i=1}^{n_{0}} \underset{E_{2} \backslash B_{m_{0}}^{a}}{ } \phi\left(t,\left\|f_{i}(t)\right\|_{X}\right) d \mu \\
& \leq \int_{E_{2} \backslash B_{m_{0}}^{a}} n_{0} \varphi\left(t, \max _{1 \leq i \leq n_{0}}\left\{\left\|f_{i}(t)\right\|_{X}\right\}\right) d \mu \\
& <\int_{E_{2} \backslash B_{m_{0}}^{a}} n_{0} \phi\left(t, \frac{f(t)}{a}\right) d \mu \\
& \leq \int_{T \backslash B_{m_{0}}^{a}} n_{0} \phi\left(t, \frac{f(t)}{a}\right) d \mu<\frac{1}{2} . \\
& \sum_{i=1}^{n_{0}} \tilde{I}_{\phi}\left(f_{i} \chi_{T \backslash\left(E_{1} \cup E_{21}\right)}\right)=\sum_{i=1}^{n_{0}} \tilde{I}_{\phi}\left(f_{i} \chi_{T \backslash E}\right)+\sum_{i=1}^{n_{0}} \tilde{I}_{\phi}\left(f_{i} \chi_{E_{22}}\right)<1 \\
& \text { because }\left\|f_{i}\right\|=1 \text { for } i=1,2, \ldots, n_{0} \text { and } \phi \in \Delta_{2} \text {, } \\
& \text { then } \tilde{I}_{\phi}\left(f_{i}\right)=1 \text { for } i=1,2, \ldots, n_{0} \text {. as a result, } \\
& \sum_{i=1}^{n_{0}} \tilde{I}_{\phi}\left(f_{i} \chi_{E_{1} \cup E_{21}}\right) \geq n_{0}-1 . \\
& \left(\frac{1}{2}\left(f_{i}-f_{j}\right) \chi_{E_{1} \cup E_{21}}\right)
\end{aligned}
$$

Obtained, 
$=\frac{1}{n_{0}}\left(\begin{array}{c}n_{0} \\ 2\end{array}\right)\left(\sum_{i=1}^{n_{0}} \tilde{I}_{\phi}\left(f_{i}\right)-\sum_{i=1}^{n_{0}} \tilde{I}_{\phi}\left(f_{i} \chi_{E_{1} \cup E_{21}}\right)\right)+\frac{r}{n_{0}}\left(\begin{array}{c}n_{0} \\ 2\end{array}\right) \sum_{i=1}^{n_{0}} \tilde{I}_{\phi}\left(f_{i} \chi_{E_{1} \cup E_{21}}\right)$

$$
\begin{aligned}
& =\left(\begin{array}{c}
n_{0} \\
2
\end{array}\right)\left(1-\frac{1-r}{n_{0}} \sum_{i=1}^{n_{0}} \tilde{I}_{\phi}\left(f_{i} \chi_{E_{1} \cup E_{21}}\right)\right) \\
& \leq\left(\begin{array}{c}
n_{0} \\
2
\end{array}\right)\left(1-\frac{(1-r)\left(n_{0}-1\right)}{n_{0}} \leq\left(\begin{array}{c}
n_{0} \\
2
\end{array}\right)(1-p)\right), \text { with } \\
& p=\frac{(1-r)}{2} .
\end{aligned}
$$

So, be found $i_{1}, j_{1} \in\left\{1,2, \ldots, n_{0}\right\}$ so that

$\tilde{I}_{\phi}\left(\frac{1}{2}\left(f_{i_{1}}-f_{j_{1}}\right)\right) \leq 1-p$.

as a result, because $\phi \in \Delta_{2}$, obtained $\left\|\frac{1}{2}\left(f_{i_{1}}-f_{j_{1}}\right)\right\| \leq 1-q(p)$, with $0<q(p)<1$.

obtained that space $L_{\phi}(\mu, X)$ P-convexs.

\section{Conclusion}

Modular $I_{\phi}(f)=\int_{T} \phi(t, f(t)) d \mu$ generates the Musielak-Orlicz function space $L_{\phi}=\left\{f \in L^{0}: I_{\phi}(c f)<\infty\right.$ for some $\left.c>0\right\}$.

Furthermore, for every Musielak - Orlicz function $\phi$, we define function $\phi^{*}(t, v)=\sup _{u>0}\{u|v|-\phi(t, u)\}$, which is also a Musielak - Orlicz function and it is called the complementary function in the sense of Young. Modular $\tilde{I}_{\phi}(f)=\int_{T} \phi\left(t,\|f(t)\|_{X}\right) d \mu$ generates the Musielak-Orlicz function spaces of Bochner type $L_{\phi}(\mu, X)=\left\{f \in L^{0}(T, X):\|f(.)\|_{X} \in L_{\phi}\right\}$. Furthermore, $L_{\phi}(\mu, X)$ is a Banach space.

The Musielak-Orlicz function spaces of Bochner type $L_{\phi}(\mu, X)$ is P-convex if and only if both $L_{\phi}$ and $X$ are P-convex. Furthermore, it is proved that reflexivity is equivalent to $\mathrm{P}$ convexity.

\section{Acknowledgement}

Acknowledgments are submitted to the Department of Mathematics,UGM for support in this research.

\section{References}

[1] W. Orlicz, Linear Functional Analysis, World Scientific, London, 1992.

[2] H. Hudzik, A. Kaminska, and W. Kurc, Uniformly non-ln ${ }^{(1)}$ Musielak-Orlicz Spaces, Bull. Acad. Polon. Sci. Math., 35, 7-8, pp. 441-448, 1987.

[3] H. Hudzik, and S. Chen, On Some Convexities of Orlicz and Orlicz-Bochner Spaces, Comment. Math., Univ. Carolinae 029 No. 1, pp. 13-29, 1988.

[4] P. Kolwicz, and R. Pluciennik, P-convexity of Musielak-Orlicz Function Spaces of Bochner Type, Revista Matematica Complutense Vol. 11 No. 1. 1998.

[5] Y. Yining, and H. Yafeng, P-convexity property in Musielak-Orlicz sequence spaces, Collect. Math. 44, pp. 307-325, 2008.

[6] D.P. Giesy, On a Convexity Condition in Normed Linear Spaces, Transactions of the American Mathematical Society Vol. 125 No. 1, pp. 114-146, 1966.

[7] P. Kolwicz, and R. Pluciennik, On P-convex Musielak-Orlicz Spaces, Comment. Math., Univ . Carolinae 36, pp. 655-672, 1995.

[8] P. Kolwicz, and R. Pluciennik, P-convexity of Bochner-Orlicz Spaces, Proc. Amer. Math. Soc.,

[9] P. Kolwicz and R. Pluciennik, P-convexity of Musielak-Orlicz Sequence Spaces of Bochner Type, Collect. Math. 1997. 